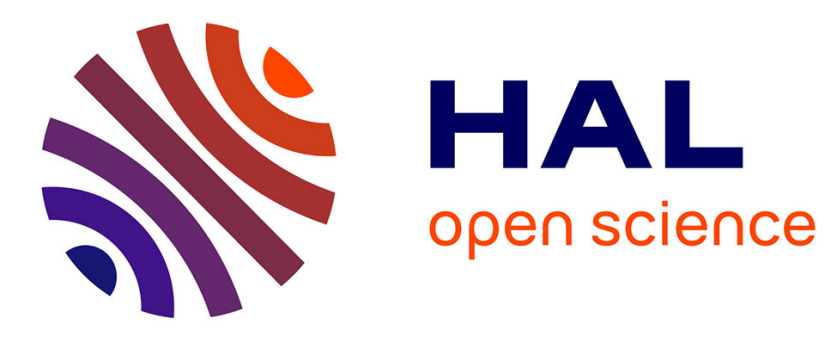

\title{
Spatiotemporal response of rat visual cortex during moving stimuli using Functional Ultrasound (fUS) Imaging
}

Marc Gesnik, Kevin Blaize, Alexandre Dizeux, José-Alain Sahel, Mathias

Fink, Thomas Deffieux, Jean-Luc Gennisson, Serge Picaud, Mickaël Tanter

\section{To cite this version:}

Marc Gesnik, Kevin Blaize, Alexandre Dizeux, José-Alain Sahel, Mathias Fink, et al.. Spatiotemporal response of rat visual cortex during moving stimuli using Functional Ultrasound (fUS) Imaging. 2016 IEEE International Ultrasonics Symposium (IUS), 2016, Tours, France. hal-03275166

\section{HAL Id: hal-03275166 https://hal.science/hal-03275166}

Submitted on 30 Jun 2021

HAL is a multi-disciplinary open access archive for the deposit and dissemination of scientific research documents, whether they are published or not. The documents may come from teaching and research institutions in France or abroad, or from public or private research centers.
L'archive ouverte pluridisciplinaire HAL, est destinée au dépôt et à la diffusion de documents scientifiques de niveau recherche, publiés ou non, émanant des établissements d'enseignement et de recherche français ou étrangers, des laboratoires publics ou privés. 


\title{
Spatiotemporal response of rat visual cortex during moving stimuli using Functional Ultrasound (fUS) Imaging
}

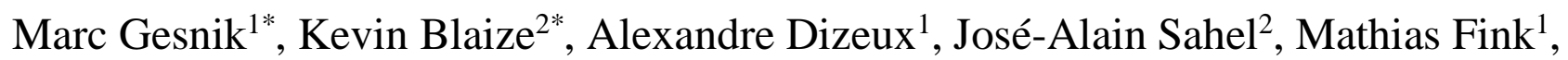 \\ Thomas Deffieux $^{1}$, Jean-Luc Gennisson ${ }^{1}$, Serge Picaud ${ }^{2 \dagger}$, Mickaël Tanter ${ }^{1 \dagger}$ \\ ${ }^{1}$ Institut Langevin (ESPCI Paris, PSL, Research University, CNRS UMR 7587, INSERM U979), Paris 75005, Paris, France \\ ${ }^{2}$ Institut de la Vision (INSERM U986), Paris, France \\ ${ }^{\dagger} \mathrm{SP}$ and MT are co-last authors. ${ }^{*} \mathrm{MG}$ and KB are co-first authors.
}

\begin{abstract}
The spatiotemporal study of the visual system of the rodent is a challenging area that conventional functional imaging modalities, despite fundamental achievements, still struggle to study nowadays. In the other hand Functional Ultrasound (fUS) offers an unprecedented combination of spatiotemporal resolution. By coupling fUS with an adapted visual stimulation device, we mapped the vision-evoked activity in the rodent brain. We then optimized the visual-stimulus toward the spatiotemporal study of the rodent visual pathway using fUS.
\end{abstract}

Keywords-functional ultrasound; vison; rodent brain, angiography

\section{INTRODUCTION}

The ability to process visual information surrounding each individual is one of the most astonishing and complex task each vertebrate can perform. This ability relies on the cerebral visual system. Its complex chain of information processing begins with the gathering of photons on the retina. The visual information travels along the optic nerves, is pre-processed in thalamic relays such as the left and right Lateral Geniculate Nuclei (LGN) or the Superior Colliculus (SC) and is finally processed in the Visual Cortex. The so-processed information is then sent to other cerebral areas that will for instance trigger an action or a memorization [1]. This complex and multi-component system has been widely studied using electrophysiology [2]-[4]. Nevertheless, due to its three dimensional spatial complexity at varying depth in the brain, its interactions with other functional regions of the brain and a variety of dynamic tasks performed along the chain, the full understanding of the visual system remains a challenge.

Although electrophysiology, fMRI [5] and optical intrinsic imaging [6], [7] have largely contributed to understand the visual system, they all suffer drawbacks. The electrophysiology, though precise and direct is local and invasive. fMRI, though $3 \mathrm{D}$, has a pour spatiotemporal resolution and need higher and higher magnetic fields to resolve the small structures involved in the rodent vision. And optical intrinsic imaging, though richly

This work was supported by the European Research Council SYNERGY Grant scheme (HELMHOLTZ, ERC Grant Agreement \#610110), Europe. resolved in time and space, does not image in depth because of light scattering in biological tissue.

In the present study, we studied the ability of functional ultrasound (fUS) imaging [8] to image the visual system. fUS is a recently developed functional neuroimaging technique based on plane waves ultrafast ultrasound imaging [9] and blood flow measurement which has already offered new insights in diagnostic imaging, blood flow imaging [10]-[12] and functional imaging [13].

By using fUS imaging and a simple visual stimulation setup we investigated the feasibility of spatiotemporally studying the visual system in the rat brain. Using a motorized translation we were able to map the activation of the visual cortex and of the Superior Colliculus and LGN along the visual pathway in 2D and in 3D. We also leveraged simple changes in the visual stimuli to assess changes in the response intensity or localization at these visual areas.

\section{METHODS}

\section{A. Animals, surgical procedures}

Adults male Long-Evans rats (Janvier Labs; France) weighting $250 \mathrm{~g}$ to $350 \mathrm{~g}$, and aged 7 to 9 weeks were included in this study. All rats underwent surgical craniotomy before the imaging session. Anaesthesia was induced with 5\% isofluorane and followed by intraperitoneal (i.p.) injection of a mixture of ketamine $(60 \mathrm{mg} / \mathrm{kg}$, Imalgene $1000 \AA$, Merial; France) and medetomidine $\left(0.4 \mathrm{mg} / \mathrm{kg}\right.$, Domitor ${ }^{\circledR}$, Pfizer, Santé Animal; France) and then was maintained by a periodic i.p. dosage using $1 / 3$ of the initial dose. Buprenorphine was injected subcutaneously (0.05 mg/kg, Buprecare $\left.{ }^{\circledR}\right)$.

All experiments were conducted in accordance with the European Council Directive (2010/63/EU) and the study was approved by the institutional and regional committees for animal care (Committee Charles Darwin $\mathrm{n}^{\circ} 5$, registration number 5486).

\section{B. Stimuli delivery}

Visual stimuli were delivered using a LCD screen $(\mathrm{P} 2314 \mathrm{H}$ 23 ", Dell), in front of the rat at $8 \mathrm{~cm}$, therefore covering $145^{\circ}$ of 

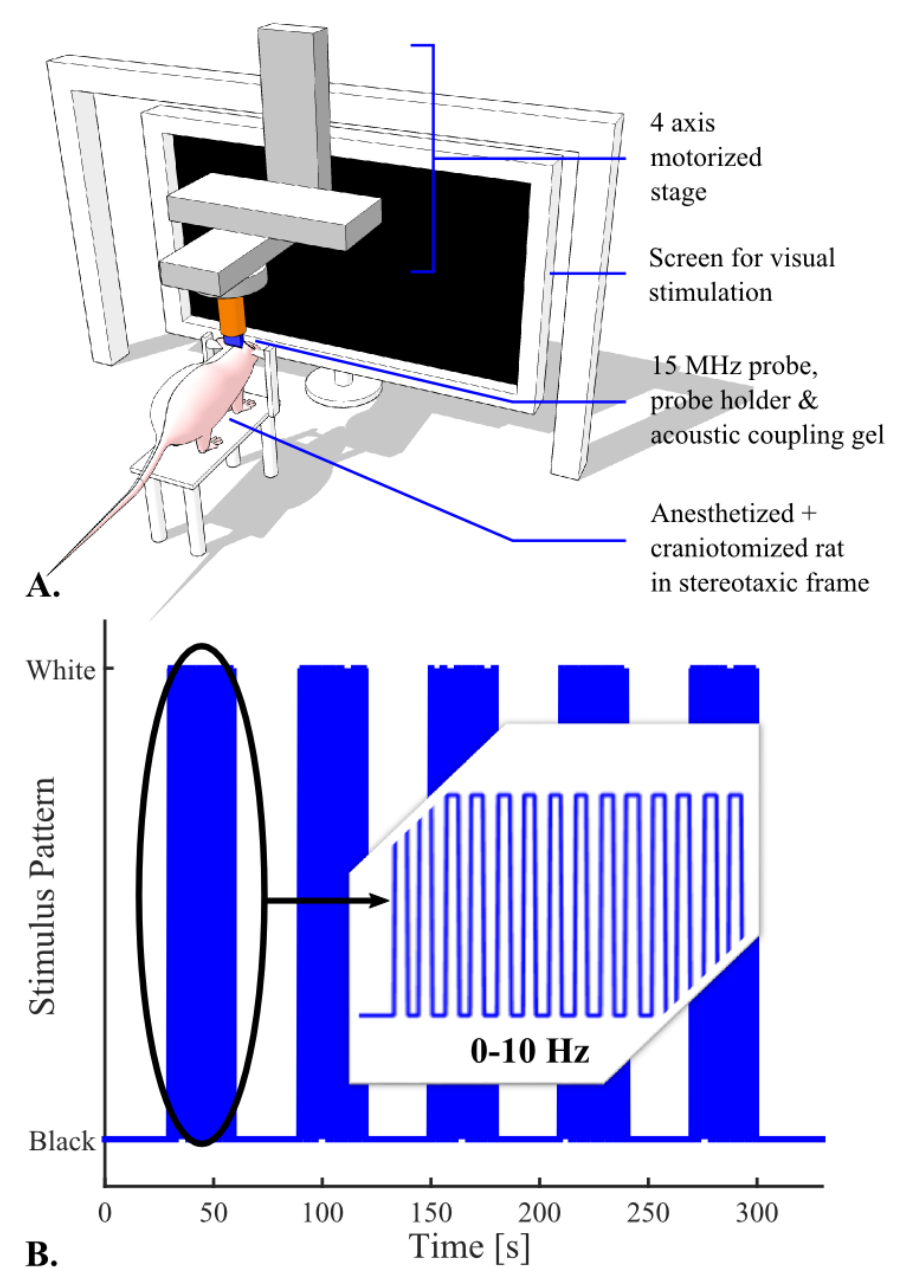

Fig. 1. A. Schematic view of the experimental setup. B. Stimulus pattern for episodic visual stimulation: $30 \mathrm{~s}$ of rest are followed with $30 \mathrm{~s}$ of flickering of the screen at a frequency in the $[0 ; 10 \mathrm{~Hz}]$

the visual field in the horizontal dimension. Stimulation runs consist in episodic presentations of $30 \mathrm{~s}$ of flickering on the screen interleaved with $30 \mathrm{~s}$ black screen period for rest (Fig. 1A and $\mathrm{B})$.

\section{C. fUS acquisition sequence and data analysis}

fUS imaging was performed using a linear ultrasound probe (128 elements, $15 \mathrm{MHz}, 110 \mu \mathrm{m}$ pitch and $8 \mathrm{~mm}$ elevation focus, Vermon; Tours, France) driven by an ultrafast ultrasound scanner (Aixplorer, Supersonic Imagine; Aix-en-Provence, France). The biophysics, technical procedure and statistical analysis for fUS were fully explained in our previous publications [13], [14].

In summary, the ultrasound sequence works as follow: First, the brain is insonified by using tilted ultrasonic plane wave [15] with a high Pulse Repetition Frequency (PRF) $(20 \mathrm{kHz})$. Second, the backscattered echoes coming from a wide-field of view on the transducer array are recorded. Finally, the raw data are beamformed to produce an image in few hundreds of ms.
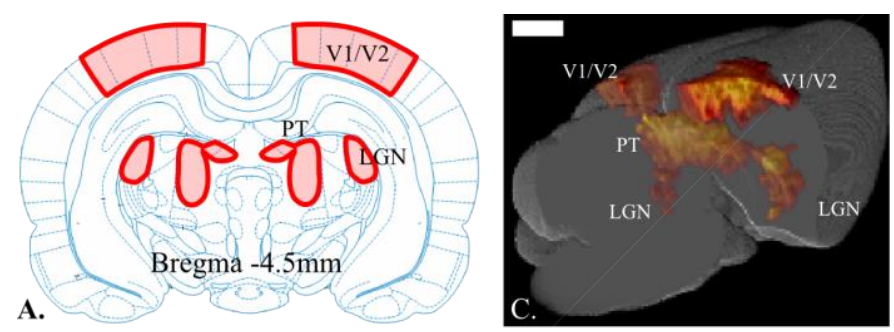

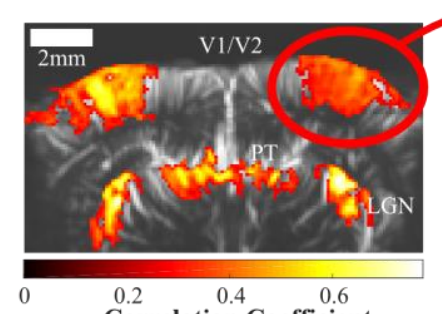

B. Correlation Coefficient

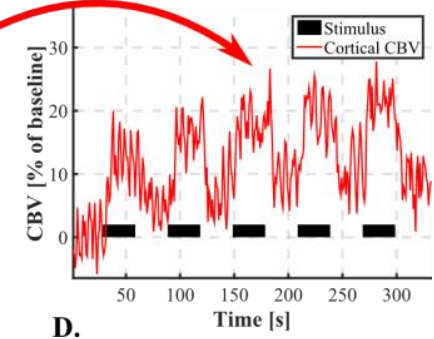

D.
Fig. 2. fUS imaging of the visual areas in the rat. A. Coronal schematic view of the rat brain at Bregma $-4.5 \mathrm{~mm}$ with the delimitations of Visual Cortex (V1/V2), Lateral Geniculate Nucleus (LGN) and Pretectal Nucleus (PT). B. fUS imaging of the vision-induced activity at Bregma $-4.5 \mathrm{~mm}$. The precision of the map allows to retrieve the shape of the 3 vision related structures present in that coronal slice. C. 3D fUS imaging of the vision induced activity in the rat brain. V1/V2, PT and LGN, which were manually superimposed to a Calabrese rat brain model. . Scale bars $=2 \mathrm{~mm}$. D Mean CBV signal over the activated pixels in the visual cortex.

The ultrasonic sequence consists in 5 to $10 \mathrm{~min}$ of continuous firing at 500 frames/s. Each frame is a coherent compounding of 11 plane waves with a $20 \mathrm{kHz}$ pulse repetition frequency. To discriminate the blood motion from tissue motion artefacts in these set of data, we used a recently developed Singular Value Decomposition (SVD) based spatiotemporal clutter filter technique [16]. Finally one image of power Doppler intensity (which is proportional to the cerebral blood volume, (CBV) [17]) is obtained by the incoherent temporal mean of the blood signal in each pixel. The continuous firing allows a temporal sampling of the CBV that can go down to 500 frames/s.

The ultrasonic probe can be translated using a motorized stage described in [18] and the fUS sequence repeated in other planes to obtain 3D fUS acquisition.

\section{RESULTS}

\section{A. 2D fUS imaging of the CBV response to a simple visual stimulus}

The ultrasonic probe was positioned over a coronal plane at Bregma $-4.5 \mathrm{~mm}$ that presents several known vision-involved structures. Those structures are, the visual cortex (V1/V2), the Lateral Geniculate Nucleus (LGN) and the Pretectal Nucleus (PT), located in the colliculi region (Fig. 2A). While imaging with fUS ultrasonic sequence, we episodically stimulated the full visual receptive field by $3 \mathrm{~Hz}$ full screen flickering during stimulation periods.

After correlation with the stimulus pattern and noise $\sigma$ thresholding, we obtained a functional activation map (Fig. 2B) that matches very nicely the expected areas. Furthermore, the 


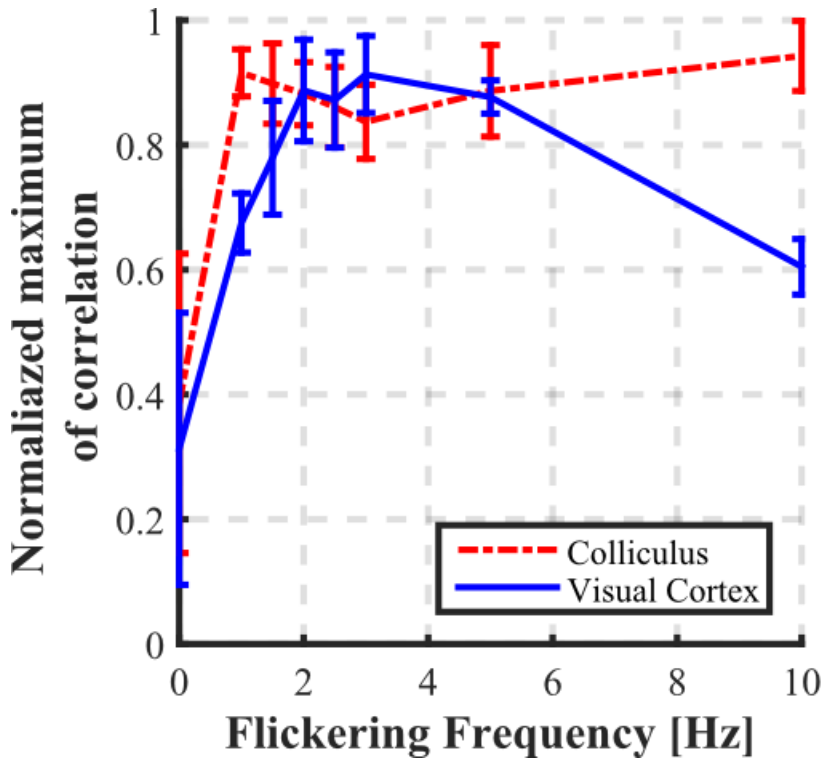

Fig. 3. Optimization of flicker parameters for fUS imaging of vision. Influence of the flickering frequency on the response amplitude in the colliculus (dashed red line) and in the visual cortex (solid blue line). The quality of response is measured via the maximum of correlation on the fUS data map. Each curve is normalized with the maximum value of correlation coefficient obtained in the corresponding brain area. Error bars: standard deviation on the normalized maximum of correlation in the considered area over the 5 trials of each parameter.

CVB profile in the visual cortex follows accurately the periods of stimulation (Fig. 2D). The operation can be repeated in several plan. Thus, staking the 2D fUS data, we retrieved the vision involved area in the rat brain in 3D (Fig. 2C).

\section{B. Optimisation of stimulus parameters}

In order to enhance the functional response for further studies we searched for an optimal flickering stimulus. The simplest parameter that we can optimized is the flickering frequency. It is simply defined by the frequency of the black/white alternation of the screen during the $30 \mathrm{~s}$ stimulation period. A flickering frequency of $0 \mathrm{~Hz}$ corresponds to a steady white screen for $30 \mathrm{~s}$. On a rat we performed repetitively the imaging session for 8 different flickering frequencies. fUS activation maps were computed and the maximum value of correlation was recovered in two different vision-involved areas: the colliculus region and the visual cortex (both hemisphere).

Fig. 3 illustrates the response amplitude for the different tested frequencies. In the visual cortex, it appeared that the best response is obtained at a $3 \mathrm{~Hz}$ flickering frequency while the higher $10 \mathrm{~Hz}$ frequency was eliciting a lower response. In the colliculus, the maximum was reached earlier at a $1 \mathrm{~Hz}$ frequency and the responses remained stable for higher frequencies up to $10 \mathrm{~Hz}$. We repeated the protocol on another rat and obtained a similar curve.

\section{Lateralization of the visual response to simple stimuli}

The visual cortex treats information coming from the contralateral visual field, for instance, the left hemisphere treating visual information from the right visual field [1]. This
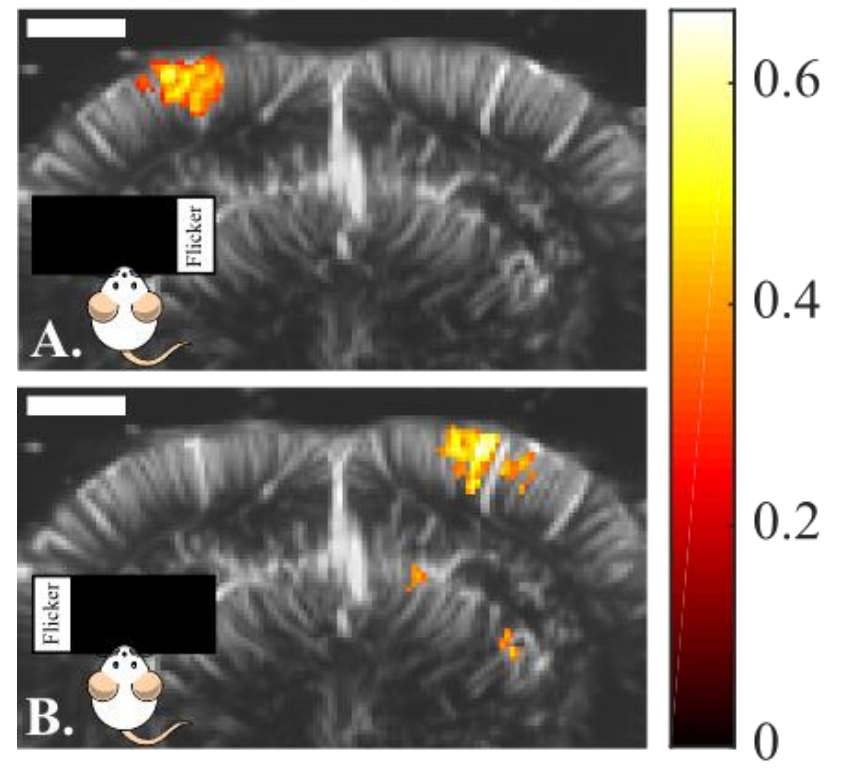

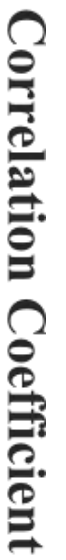

Fig. 4. Visualization by fUS of the lateralization of the visual response in the visual cortex: A. A stimulus in the right part of the visual field results in a CBV increase in the left visual cortex, when (B.) a stimulus in the left part of the visual field results in a CBV increase in the right visual cortex. Scale bar = $2 \mathrm{~mm}$.

lateralized response can thus serves as an additional confirmation that fUS imaging observations are not mere artefacts but true measurements of visual circuit activity. To address this question the ultrasonic probe was positioned over a coronal slice containing the visual cortex in both hemispheres. The flickering frequency was fixed at $3 \mathrm{~Hz}$. However, instead of stimulating the complete visual field using the whole screen, the screen was divided in 5 column sectors of identical width. In the first imaging session, only the extreme-right sector was flickering, whereas in the second time, we shifted the illumination to the extreme-left sector.

Fig. 4 illustrates the results of those 2 imaging sessions. Each sector is producing a fUS signal only in the respective contralateral visual cortex. These observations demonstrate the ability of the fUS imaging set-up to discriminate the projection of spatially distinct stimuli in the brain consistent with the visual processing lateralization.

\section{DISCUSSION AND CONCLUSION}

In the present study fUS imaging was used on anesthetized rats to perform several basics functional imaging studies related to vision. The high resolution and 3D mapping of visual patterns in the whole brain such as lateralized stimulus and flickering stimulus was presented. The best stimulation parameters for imaging of the visual cortex were found to be a $3 \mathrm{~Hz}$ flickering frequency in accordance with the literature [19].

Compared to BOLD fMRI, the fUS technique benefits from a high spatial and temporal resolution at low cost with a portable device. Compared to optical imaging techniques which provides high resolution functional imaging but are limited to the superficial cortical areas, fUS can be used to image the full depth of the brain, including the LGN or the colliculus in our case. 
It has been widely demonstrated through different functional imaging methods [20] that the treatment of the visual information is spatialized in the rodent brain. By stimulating the visual field with a different kind of stimuli, we showed that it is possible to study visual spatialization in the brain with fUS. As in [6], the screen was divided in different sectors and successively used to stimulate the rat visual field by using those different sectors to infer the corresponding activated regions in the brain.

\section{REFERENCES}

[1] J. Nolte, The Human Brain: An Introduction to Its Functional Anatomy. 2009.

[2] A. D. Adams and J. M. Forrester, "the Projection of the Rat'S Visual Field on the Cerebral Cortex," Q J Exp Physiol Cogn Med Sci, vol. 53, pp. 327336, 1968.

[3] S. G. Espinoza, H. C. Thomas, B. Y. D. H. Hubel, a D. T. N. Wiesel, D. H. N. Hubel, T. N. Wiesel, B. Y. D. H. Hubel, and a D. T. N. Wiesel, "Retinotopic organization of striate and extrastriate visual cortex in the hooded rat," Brain Res., vol. 160, no. 1, pp. 106-154, 1983.

[4] B. Y. D. H. Hubel, a D. T. N. Wiesel, D. N. Hubel, T. N. Wiesel, B. Y. D. H. Hubel, and a D. T. N. Wiesel, "AND FUNCTIONAL ARCHITECTURE IN THE CAT' S VISUAL CORTEX From the Neurophysiolojy Laboratory, Department of Pharmacology central nervous system is the great diversity of its cell types and inter- receptive fields of a more complex type ( Part I ) and to ,"Most, vol. 160, pp. 106$154,1962$.

[5] J. Warnking, M. Dojat, a Guérin-Dugué, C. Delon-Martin, S. Olympieff, N. Richard, a Chéhikian, and C. Segebarth, "fMRI retinotopic mapping-step by step.," Neuroimage, vol. 17, no. 4, pp. 1665-1683, 2002.

[6] C. Gias, N. Hewson-Stoate, M. Jones, D. Johnston, J. E. Mayhew, and P. J. Coffey, "Retinotopy within rat primary visual cortex using optical imaging," Neuroimage, vol. 24, no. 1, pp. 200-206, 2005.

[7] V. a. Kalatsky and M. P. Stryker, "New paradigm for optical imaging: Temporally encoded maps of intrinsic signal," Neuron, vol. 38, no. 4, pp. 529-545, 2003.

[8] E. Macé, G. Montaldo, I. Cohen, M. Baulac, M. Fink, and M. Tanter, "Functional ultrasound imaging of the brain.," Nat. Methods, vol. 8, no. 8, pp. 662-4, Aug. 2011.

[9] M. Tanter and M. Fink, "Ultrafast imaging in biomedical ultrasound.," IEEE Trans. Ultrason. Ferroelectr. Freq. Control, vol. 61, no. 1, pp. 10219, Jan. 2014
[10] J. Udesen, F. Gran, K. L. Hansen, J. A. Jensen, C. Thomsen, and M. B. Nielsen, "High frame-rate blood vector velocity imaging using plane waves: Simulations and preliminary experiments," IEEE Trans. Ultrason. Ferroelectr. Freq. Control, vol. 55, no. 8, pp. 1729-1743, 2008.

[11] J. Bercoff, G. Montaldo, T. Loupas, D. Savery, F. M??zi??re, M. Fink, and M. Tanter, "Ultrafast compound doppler imaging: Providing full blood flow characterization," IEEE Trans. Ultrason. Ferroelectr. Freq. Control, vol. 58, no. 1, pp. 134-147, 2011.

[12] B.-F. Osmanski, M. Pernot, G. Montaldo, A. Bel, E. Messas, and M. Tanter, "Ultrafast Doppler imaging of blood flow dynamics in the myocardium.," IEEE Trans. Med. Imaging, vol. 31, no. 8, pp. 1661-8, Aug. 2012.

[13] B. F. Osmanski, C. Martin, G. Montaldo, P. Lanièce, F. Pain, M. Tanter, and H. Gurden, "Functional ultrasound imaging reveals different odorevoked patterns of vascular activity in the main olfactory bulb and the anterior piriform cortex.," Neuroimage, vol. 95, pp. 176-84, Jul. 2014.

[14] E. Mace, G. Montaldo, B.-F. Osmanski, I. Cohen, M. Fink, and M. Tanter, "Functional ultrasound imaging of the brain: theory and basic principles.," IEEE Trans. Ultrason. Ferroelectr. Freq. Control, vol. 60, no. 3, pp. 492506, Mar. 2013

[15] G. Montaldo, M. Tanter, J. Bercoff, N. Benech, and M. Fink, "Coherent plane-wave compounding for very high frame rate ultrasonography and transient elastography," IEEE Trans. Ultrason. Ferroelectr. Freq. Control, vol. 56, no. 3, pp. 489-506, 2009.

[16] C. Demene, T. Deffieux, M. Pernot, B.-F. Osmanski, V. Biran, S. Franqui, J.-M. Correas, I. Cohen, O. Baud, and M. Tanter, "Spatiotemporal clutter filtering of ultrafast ultrasound data highly increases Doppler and fUltrasound sensitivity.," IEEE Trans. Med. Imaging, vol. PP, no. 99, p. $1,2015$.

[17] J. M. Rubin, R. O. Bude, P. L. Carson, R. L. Bree, and R. S. Adler, "Power Doppler US: a potentially useful alternative to mean frequency-based color Doppler US.," Radiology, vol. 190, no. 3, pp. 853-856, 1994.

[18] C. Demené, E. Tiran, L. A. Sieu, A. Bergel, J. L. Gennisson, M. Pernot, T. Deffieux, I. Cohen, and M. Tanter, "4D microvascular imaging based on ultrafast Doppler tomography," Neuroimage, vol. 127, pp. 472-483, 2015 .

[19] C. J. Bailey, B. G. Sanganahalli, P. Herman, H. Blumenfeld, A. Gjedde, and F. Hyder, "Analysis of Time and Space Invariance of BOLD Responses in the Rat Visual System.," Cereb Cortex, vol. 23, no. 1, pp. 210-22, 2013.

[20] S. G. Espinoza, J. E. Subiabre, and H. C. Thomas, "Retinotopic organization of striate and extrastriate visual cortex in the golden hamster (Mesocricetus auratus).," vol. 25, no. 2, pp. 101-107, 1992. 